\title{
Avaliação tecnológica de variedades de cana-de-açúcar influenciadas por diferentes adubações e supressões de irrigação ${ }^{1}$
}

\author{
Franklin Meireles de Oliveira ${ }^{2}$, Ignacio Aspiazü ${ }^{3}$, Marcos Koiti Kondo ${ }^{4}$, Iran Dias Borges 5 , \\ Rodinei Facco Pegoraro ${ }^{6}$, Edilson Jardim Vianna
}

\begin{abstract}
RESUMO
Objetivou-se neste trabalho avaliar as características tecnológicas de duas variedades de cana-de-açúcar submetidas a diferentes níveis de adubação e supressões de irrigação. O experimento foi instalado na Fazenda Experimental da Universidade Estadual de Montes Claros, em Janaúba-MG. O delineamento utilizado foi o de blocos casualizados, três repetições, em parcelas subdivididas ( x 3 × 6), sendo na parcela duas variedades de cana-de-açúcar, RB85-5453 e SP80-1816, com três épocas de supressão de irrigação e precipitação após emergência dos perfilhos (DIAC), caracterizadas aos 165, 195 e 225 dias, e na subparcela adubou-se com seis níveis de adubação mineral, $1\left(0 \mathrm{~kg} \mathrm{ha}^{-1}\right.$ de N e $\left.\mathrm{K}_{2} \mathrm{O}\right)$, 2 (14 kg ha ${ }^{-1}$ de Ne $\left.33 \mathrm{~kg} \mathrm{ha}^{-1} \mathrm{de} \mathrm{K}_{2} \mathrm{O}\right), 3$ (29 kg ha-1 de Ne $\left.66 \mathrm{~kg} \mathrm{ha}^{-1} \mathrm{de} \mathrm{K}_{2} \mathrm{O}\right), 4\left(43 \mathrm{~kg} \mathrm{ha}^{-1} \mathrm{de} \mathrm{Ne}_{100 \mathrm{~kg} \mathrm{ha}} \mathrm{ke} \mathrm{K}_{2} \mathrm{O}\right)$, 5 (57 kg ha-1 de Ne $133 \mathrm{~kg} \mathrm{ha}^{-1}$ de $\left.\mathrm{K}_{2} \mathrm{O}\right)$ e $6\left(71 \mathrm{~kg} \mathrm{ha}^{-1}\right.$ de Ne $166 \mathrm{~kg} \mathrm{ha}^{-1} \mathrm{de} \mathrm{K}_{2} \mathrm{O}$. As variáveis avaliadas foram: Brix, Pol do caldo, Açúcar Total Recuperável (ATR), Pureza e Litros de álcool estimados. Os níveis de adubação influenciaram a qualidade do caldo, obtendo média geral de 24,30 brix, 20,976\% pol do caldo, com a variedade RB85-5453 obtendo maiores valores para todas as variáveis analisadas, a variedade RB85-5453 apresentou maiores teores de ATR, atingindo valores superiores a 189,25 $\mathrm{kg} \mathrm{t}^{-1}$. Observaram-se valores em álcool estimados máximo de $115,09 \mathrm{~L} \mathrm{t}^{-1}$ da variedade RB85-5453 e mínimo de 90,21 L t t-1 na SP80-1816. Com aplicação de $71 \mathrm{~kg} \mathrm{ha}^{-1}$ de $\mathrm{Ne} 166 \mathrm{~kg} \mathrm{ha}^{-1}$ de $\mathrm{K}_{2} \mathrm{O}$ de adubação obteve-se melhor desempenho nas condições de realização deste trabalho. Entre as duas variedades estudadas a RB85-5453 é a mais indicada para a região, pois atingiu altos níveis em produção industrial.
\end{abstract}

Palavras-chave: análises industriais, fertilização, semiárido, disponibilidade hídrica.

\section{ABSTRACT \\ Technology assessment of sugarcane varieties influenced by different fertilization and water stress}

The objective of this study was to evaluate the technological characteristics of two varieties of cane sugar under different levels of fertilization and irrigation suppression. The experiment was conducted at the Experimental Farm of the State University of Montes Claros, in Janaúba-MG. The experimental design was randomized blocks, three replications in a split plot ( 2 × 3 × 6), and in part two varieties of cane sugar, RB85-5453 and SP80-1816, with three irrigation season suppression and precipitation after emergency of tiller (DIAC), characterized at 165, 195 and 225 days, and the subplot

Recebido para publicação em 26/04/2011 e aprovado em 26/09/2012.

${ }^{1}$ Parte da dissertação de Mestrado do primeiro autor, apresentada ao Programa de Pós-Graduação em Produção Vegetal no Semiárido do Instituto de Ciências Agrárias da Universidade Estadual de Montes Claros. Fonte financiadora FAPEMIG.

${ }^{2}$ Tecnólogo em Produção de Cachaça, Mestre. Departamento de Ciências Agrárias, Universidade Estadual de Montes Claros, Rua Gão Mogol, 474, Vila Formosa, 39550-000, Taiobeiras, Minas Gerais, Brasil. franklin.meireles@yahoo.com.br (autor para correspondência).

${ }^{3}$ Engenheiro-Agrônomo, Doutor. Departamento de Ciências Agrárias, Universidade Estadual de Montes Claros, Av. Reinaldo Viana, 2.630 Caixa Postal 91, Bico da Pedra -39440-000 Janaúba, Minas Gerais, Brasil. aspiazu@unimontes.br

${ }^{4}$ Engenheiro-Agrônomo, Doutor. Departamento de Ciências Agrárias, Universidade Estadual de Montes Claros, Av. Reinaldo Viana, 2.630 Caixa Postal 91, Bico da Pedra -39440-000 Janaúba, Minas Gerais, Brasil. (Bolsista BIPDT da FAPEMIG). marcos.kondo@unimontes.br

${ }^{5}$ Engenheiro-Agrônomo, Doutor. Departamento de Ciências Agrárias, Universidade Federal de São João Del-Rei, Campus Sete Lagoas, Rodovia MCJ 424, Km 47, Caixa Postal 56, 35701970, Sete Lagoas, Minas Gerais, Brasil. idb@ufsj.edu.br

${ }^{6}$ Engenheiro-Agrônomo, Doutor. Departamento de Ciências Agrárias, Universidade Estadual de Montes Claros, Av. Reinaldo Viana, 2.630 Caixa Postal 91, Bico da Pedra -39440-000 Janaúba, Minas Gerais, Brasil (Bolsista BIPDT da FAPEMIG. rodinei_pegoraro@yahoo.com.br

Engenheiro de Alimentos. Laboratório Industrial da Usina São Judas Tadeu (SADA Bioenergia e Agricultura), Município de Jaíba, Minas Gerais. edilson@sadabioenergia.com.br 
with six levels of mineral fertilizer, $1\left(0 \mathrm{~kg} \mathrm{ha}^{-1}\right.$ of $\mathrm{N}$ and $\left.\mathrm{K}_{2} \mathrm{O}\right), 2\left(14 \mathrm{~kg} \mathrm{ha}^{-1} \mathrm{~N}\right.$ and $\left.33 \mathrm{~kg} \mathrm{ha}^{-1} \mathrm{~K}_{2} \mathrm{O}\right), 3\left(29 \mathrm{~kg} \mathrm{ha}^{-1} \mathrm{~N}\right.$ and 66 $\left.\mathrm{kg} \mathrm{ha}^{-1} \mathrm{~K}_{2} \mathrm{O}\right), 4\left(43 \mathrm{~kg} \mathrm{ha}^{-1}\right.$ and $100 \mathrm{~kg} \mathrm{~N} \mathrm{ha}^{-1}$ of $\left.\mathrm{K}_{2} \mathrm{O}\right), 5\left(57 \mathrm{~kg} \mathrm{ha}^{-1}\right.$ and $\left.133 \mathrm{~kg} \mathrm{~N} \mathrm{ha}^{-1} \mathrm{~K}_{2} \mathrm{O}\right)$ and $6\left(71 \mathrm{~kg} \mathrm{~N} \mathrm{ha}^{-1}\right.$ and $166 \mathrm{~kg}$ $\mathrm{ha}^{-1}$ of $\mathrm{K}_{2} \mathrm{O}$. The variables evaluated were: Brix, Pol of juice, Total Recoverable Sugars (ATR), Purity and liters of alcohol estimated. Nutrient levels influenced the quality of the juice, achieving overall average of $24.30^{\circ}$ brix, pol $20.976 \%$ of the juice. Variety RB85-5453 achieved the highest values for all variables; variety RB85-5453 showed the highest levels of ATR, reaching values higher than $189.25 \mathrm{~kg} \mathrm{t}^{-1}$. Estimated values of ethanol were maximum at $115.09 \mathrm{Lt}^{-1}$ for the variety RB85-5453 and minimum at $90.21 \mathrm{Lt}^{-1}$ for variety SP80-1816. The application of $71 \mathrm{~kg} \mathrm{ha}^{-1} \mathrm{~N}$ and $166 \mathrm{~kg} \mathrm{ha}^{-1}$ of $\mathrm{K}_{2} \mathrm{O}$ gave the best performance in the conditions of this study. Variety RB85-5453 was shown the most appropriate for the region because it reached high levels in industrial production.

Key words: industrial analyses, fertilization, semiarid, water availability.

\section{INTRODUÇÃO}

A cultura da cana-de-açúcar é considerada, atualmente, uma realidade no quesito energético, valorizada pela sua capacidade de gerar energia limpa e renovável. Em nível mundial, começa a haver uma corrida por combustíveis mais 'limpos'; entretanto, quando o assunto é expandir as fronteiras agrícolas para a produção de biocombustíveis, temem-se os seus reflexos na diminuição de áreas para a produção de alimentos. Contudo, é uma das grandes vantagens para o Brasil, país de dimensões continentais, dispondo, ainda, de terras para a expansão de cultivo da cana-de-açúcar, além de renovação na extração de etanol, um biocombustível limpo em relação aos combustíveis fósseis.

Em algumas regiões do País, especificamente no Norte de Minas Gerais, há interesse em se disseminar e modernizar a produção de cada de açúcar, aumentando as produtividades agrícola e industrial, com melhorias na qualidade da matéria-prima. Nesse sentido, os produtores mineiros têm altos investimentos em irrigação e adubação. Considerando a escassez de recursos hídricos, um dos problemas da expansão da agricultura irrigada no Estado, inclusive no setor sucroalcooleiro, é imprescindível e urgente se estudar práticas de manejo mais eficientes do uso de água. Igualmente importante é se identificar os principais problemas de ordem nutricional das plantas em cada área de produção.

Na literatura, são várias as informações sobre demanda hídrica da cana-de-açúcar. Segundo Rodrigues (1995), a cultura exige entre 1.500 e $2.500 \mathrm{~mm}^{-1}$. Estudando os efeitos da irrigação sobre a qualidade da cana CP 65-357, submetida a três níveis de irrigação $(99,85$ e $65 \%$ da fração de esgotamento da água do solo), no Estado do Texas, Wiedenfeld (1995) obteve rendimentos em açúcar de 13, 10 e $7,5 \mathrm{t} \mathrm{ha}^{-1}$, respectivamente, sem variar sua pureza (87, 86 e $85 \%)$. Posteriormente, Wiedenfeld (2000) avaliou diferentes níveis de estresse hídrico na cultura, em cinco diferentes estádios de desenvolvimento, registrando reduções de $11 \%$ no rendimento em açúcar quando a cultura foi submetida ao estresse no período entre 257 e 272 dias após o plantio e de $19 \%$ quando o estresse hídrico ocorreu entre 302 e 347 dias após o plantio. Dantas Neto et al. (2006) testaram lâminas de irrigação variando entre 807 e 1.343 mm na cultura de cana-de-açúcar e verificaram efeito linear positivo sobre as variáveis de crescimento e quadrático para sacarose (Pol), atingindo valor de 18,1\% quando as plantas foram irrigadas com $1.125 \mathrm{~mm}$. Moura et al. (2005) observaram diferenças em ${ }^{\circ}$ Brix, Pol do caldo e Pol da cana em estudos, comparando a cana irrigada com a de sequeiro, com os valores aumentando 5,00; 10,79; e $8,63 \%$, respectivamente, quando houve irrigação.

Dentro desse enfoque, procurando atender às necessidades atuais de manejo, o presente trabalho teve por objetivo estudar o comportamento de duas variedades comerciais de cana-de-açúcar quanto às características tecnológicas influenciadas por diferentes épocas de supressão de irrigação e distintos níveis de adubação nas condições edafoclimáticas da região Norte do Estado de Minas Gerais.

\section{MATERIAL E MÉTODOS}

O experimento foi conduzido na Fazenda Experimental da Universidade Estadual de Montes Claros, em Janaúba$\mathrm{Mg}$, que possui as coordenadas geográficas $43^{\circ} 16^{\prime} 18,2^{\prime \prime}$ W e $15^{\circ} 49^{\prime} 51,5^{\prime \prime} \mathrm{S}$ e altitude de $540 \mathrm{~m}$, situada no perímetro irrigado da Associação dos Irrigantes da Margem Esquerda do Rio Gorutuba (ASSIEG). O solo da área experimental foi classificado como Latossolo Vermelho distrófico, textura média (LVd) (Embrapa, 1999). O delineamento utilizado foi o de blocos casualizados, com três repetições, em parcelas subdivididas ( $2 \times 3 \times 6)$, sendo utilizadas na parcela duas variedades de cana-de-açúcar (RB85-5453 e SP80-1816) e três épocas distintas de supressão de irrigação) $(165,195$ e 225 dias e precipitação após emergência dos perfilhos 
(DIAC) e seis níveis de adubação. Utilizou-se o sistema de irrigação com aspersores de baixa pressão.

A área era constituída de cana soca de segundo corte e o plantio foi realizado com média de 18 gemas por metro linear. Cada parcela foi composta por $126 \mathrm{~m}^{2}$, dividida em seis subparcelas, sendo cada uma de quatro linhas de $5 \mathrm{~m}$ de comprimento, espaçadas de 1,40 m. As duas linhas centrais foram consideradas como úteis para efeito de coleta de dados e observações, descartando-se $2 \mathrm{~m}$ em cada extremidade da linha.

A lâmina de irrigação foi determinada levando-se em consideração a precipitação e eficiência do sistema utilizado, bem como a evapotranspiração da cultura (ETc e Kc da cana-de-açúcar) (Bernardo et al., 2005), dispensandoa na ocorrência de precipitação. Os dados climáticos foram obtidos na estação meteorológica da Epamig, em Nova Porteirinha-MG.

Na subparcela adubou-se de uma só vez utilizando seis níveis de adubação mineral: $1\left(0 \mathrm{~kg} \mathrm{ha}^{-1}\right.$ de $\left.\mathrm{Ne} \mathrm{K}_{2} \mathrm{O}\right)$, 2 (14 kg ha-1 de Ne $\left.33 \mathrm{~kg} \mathrm{ha}^{-1} \mathrm{de} \mathrm{K}_{2} \mathrm{O}\right), 3$ (29 kg ha-1 de Ne $66 \mathrm{~kg} \mathrm{ha}^{-1} \mathrm{de} \mathrm{K}_{2} \mathrm{O}$ ), 4 (43 kg ha-1 de Ne $100 \mathrm{~kg} \mathrm{ha}^{-1}$ de $\mathrm{K}_{2} \mathrm{O}$ ), $5\left(57 \mathrm{~kg} \mathrm{ha}^{-1} \mathrm{de} \mathrm{Ne} 133 \mathrm{~kg} \mathrm{ha}^{-1} \mathrm{de} \mathrm{K}_{2} \mathrm{O}\right)$ e $6\left(71 \mathrm{~kg} \mathrm{ha}^{-1} \mathrm{de} \mathrm{N}\right.$ e $166 \mathrm{~kg} \mathrm{ha}^{-1}$ de $\mathrm{K}_{2} \mathrm{O}$ ).

As variáveis da cultura analisadas foram: quantidade de sólidos solúveis totais (Brix), Pol do caldo, Açúcares Totais Recuperáveis (ATR), Pureza e Litros de álcool estimados. Foram feitas duas coletas de dados em todas as parcelas, no mês de agosto (300 dias após emergência) e a segunda em setembro (330 dias após corte) de 2010, com determinações realizadas no laboratório Industrial da usina São Judas Tadeu (SADA Bioenergia e Agricultura), no município de Jaíba-MG. Foram coletados aleatoriamente três colmos inteiros em cada subparcela e transportados no transcorrer de $1 \mathrm{~h}$ para o laboratório, simulando o tempo real de colheita (Consecana, 2006).

Análises de Brix e Pol do caldo foram feitas pelo método da prensa hidráulica. A partir do caldo foi feita a análise do Brix (refratométrico) e determinada por métodos sacarimétricos (polarímetros ou sacarímetros). O Pol\% caldo foi obtido pela fórmula Pol\% Caldo $=(1,0078 \times$ leit. sacar. + 0,0444) x $(0,2607-0,009882 \times$ Brix $)$. A massa do bagaço, também chamada de bolo úmido (P.B.U.), foi determinada em balança eletrônica. A partir dos resultados de leitura Sacarimétrica, Brix e PBU foram obtidos os resultados de Pol da cana, Fibra, Pureza, Açúcar Redutor (AR), Brix da cana, Umidade, Açúcar Total Recuperável (ATR) e Açúcar Redutor Total (ART). Os valores de tais variáveis foram obtidas com os seguintes cálculos: Fibra $=(0,08 \times$ PBU $)+0,876$, de acordo com Fernandes (2000), C $=(1,0313-0,00575 \times$ FIBRA $)$, e o coeficiente " $C$ " representa a transformação em todo o caldo extraído; ou seja, todo caldo proveniente da prensa hidráulica. Posteriormente, encontra-se o Brix \% cana $=$ Brix do caldo $\mathrm{x}(1-$
0,01) $\mathrm{x}$ C e Pol\% cana $=$ Pol no caldo $\mathrm{x}(1-0,01 \times$ FIBRA $) \times$ C. A pureza foi obtida pela fórmula: $\mathrm{PUREZA}=(\mathrm{Pol} \%$ cana)/(Brix\% cana) x 100. O cálculo dos açúcares redutores no caldo foi feito pela fórmula: $\mathrm{AR} \%$ caldo $=(3,641-$ $0,0343 \times$ PUREZA); a AR \% cana $=$ AR no caldo $\times(1-0,01$ x FIBRA) x C (Consecana, 2006).

Os açúcares redutores totais foram determinados pela seguinte equação: ART\% caldo $=($ Pol no caldo/0,95 $)+$ AR e ART\% cana $=$ ART do caldo $\times(1-0,01 \times$ FIBRA $) \times$ C. Conhecendo-se a Pol da cana (PC) e os açúcares redutores da cana (ARC), o açúcar total recuperável é calculado pela equação: $\mathrm{ATR}=10$ x PC x 1,05263 x 0,905 + 10 x ARC x 0,905, em que: 10 x PC = pol por tonelada de cana; 1,05263 = coeficiente estequiométrico para a conversão da sacarose em açúcares redutores; e 0,905 = coeficiente de recuperação, para uma perda industrial de 9,5\% e $10 \mathrm{x}$ ARC $=$ açúcares redutores por tonelada de cana (Consecana, 2006).

A produção de álcool hidratado foi estimada pelo cálculo: ART\% caldo x 10 x 0,6475=100\% de álcool, considerando um processo fermentativo de $85 \%$ de eficiência.

A distribuição da precipitação no experimento ao longo do ciclo fenológico da cultura (360 dias), em intervalos quinzenais, encontra-se na Figura 1. As quantidades de água aplicada por meio da irrigação em cada período correspondente as supressões; L1 (165 DIAC) $=918 \mathrm{~mm}, \mathrm{~L} 2$ $(195$ DIAC $)=1.242 \mathrm{~mm}$ e L3 $(225$ DIAC $)=1.539 \mathrm{~mm}$. A precipitação aproveitável (Pap) acumulada no mesmo período foi de 873,8; 891,2; e 902,4 mm, respectivamente, resultando em quantidades totais de água aplicadas para os três DIACs distintos de L1 + Pap ( 165 DIAC) = 1.791,8mm, L2 + Pap (195 DIAC) $=2.133,2$ mm e L3 + Pap $(225$ DIAC $)=2.441,4 \mathrm{~mm}($ Figura 1$)$.

Foi feita a média das duas coletas de dados, a qual foi submetida à análise de variância e, quando houve diferença significativa pelo teste $\mathrm{F}$, realizou-se a comparação das médias pelo teste de Scott Knott $(\mathrm{p}<0,05)$ para todas as variáveis estudadas, utilizando-se do programa software SISVAR (Ferreira, 2000).

\section{RESULTADOS E DISCUSSÃO}

Verifica-se na Tabela 1 que há interações entre variedades, DIAC e níveis de adubação, tendo a variedade RB85-5453 valores maiores para todas as variáveis analisadas (Tabelas 2, 3, 4 e 5), o que pode ser explicado pela sua maturação precoce. As variedades apresentaram média geral de $24,30{ }^{\circ}$ Brix, $17,593 \%$ Pol do caldo e $85 \%$ em Pureza (Tabela 1). Os atributos de qualidade da matériaprima são os mais importantes para a indústria canavieira, visto que vão definir os rendimentos em açúcar e álcool.

Constata-se na Tabela 2 que as variedades diferiram estatisticamente na variável Brix em todos os tratamentos, com a variedade RB85-5453 superando em valores a 
SP80-1816. Nos tratamentos da variedade RB85-5453 não houve diferenças entre DIAC, $\log 0$ as diferentes supressões de irrigação não interferiram na porcentagem de sólidos solúveis acumulados na variedade, porém na SP801816 houve diferenças entre os DIACs dentro de cada nível de adubação com os menores valores encontrados nos menores DIAC (Tabela 2). A água disponível para a planta é essencial, uma vez que promove a síntese, o acúmulo e a translocação da sacarose nos colmos, podendo os produtores manipular a irrigação visando à melhoria da maturação. No trabalho realizado, a supressão de irrigação demonstra a importância da água na qualidade da planta cana-de-açúcar.
A característica Pol do caldo obteve superioridade na variedade RB85-5453 em relação à SP80-1816 com os DIAC diferindo nos tratamentos de níveis de adubo 1, 2, 3 e 4 (Tabela 5). Observou-se que a variedade RB85-5453 indicou comportamento em maturidade, podendo ser colhida precocemente por apresentar maiores acúmulos de sacarose no mesmo momento do ciclo da variedade SP801816. Por outro lado, a variedade SP80-1816, com menores índices de acúmulo de sacarose no seu colmo, é adequada para colheita médio-tardia, tendo diferenças entre supressões, valores de qualidade maiores aos 225 DIAC (Tabela 5). Todavia, segundo Araújo (2006), esse cultivar é usado no Estado de São Paulo e regiões vizinhas para

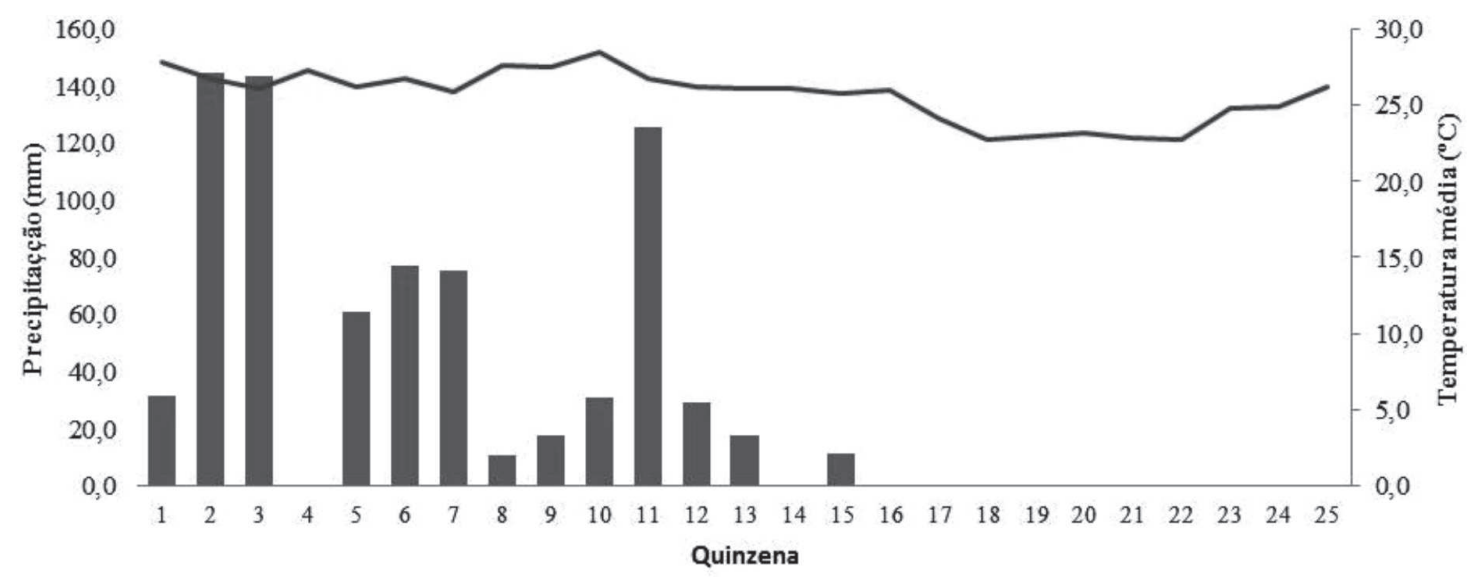

Precipitação - Temperatura Média

Figura 1. Dados médios de temperatura e precipitação pluvial acumulada por quinzena, em milímetros (mm), em Janaúba-MG, de 1\% 10/2009 a 30/09/2010. Dados obtidos na Estação Climatológica da EPAMIG, Nova Porteirinha-MG, 2011.

Tabela 1. Resumo das análises de variância para variáveis Pureza (PUR), Brix, Pol do caldo, Açúcares teórico recuperáveis (ATR), Estimativa de álcool e açúcares redutores (AR)

\begin{tabular}{|c|c|c|c|c|c|c|}
\hline \multirow[b]{2}{*}{ F.V. } & \multirow[b]{2}{*}{ G.L } & \multicolumn{5}{|c|}{ QUADRADO MÉDIO } \\
\hline & & $\begin{array}{l}\text { PUR } \\
(\%)\end{array}$ & $\begin{array}{r}\text { BRIX } \\
(\%)\end{array}$ & $\begin{array}{c}\text { POL DO } \\
\text { CALDO }(\%)\end{array}$ & $\begin{array}{l}\text { ATR } \\
\operatorname{Ktg~t}^{-1}\end{array}$ & $\begin{array}{c}\text { ÁLCOOL } \\
(\mathrm{L})\end{array}$ \\
\hline BLOCO & 2 & 0,008 & 0,560 & 0,022 & 7,32 & 4,714 \\
\hline VAR & 1 & $419,92 *$ & $150,92 *$ & $228,028 *$ & $10583,892 *$ & $3416,51 *$ \\
\hline DIAC & 2 & 6,520 & $7,47 *$ & $5,286^{*}$ & $419,7014 *$ & $93,394 *$ \\
\hline VAR*DIAC & 2 & 14,586 & $3,51 *$ & 1,561 & $286,7941 *$ & $106,940 *$ \\
\hline erro 1 & 10 & 6,324 & 0,795 & 0,676 & 69,027 & 22,191 \\
\hline NÍV & 5 & $34,928 *$ & 0,706 & $2,874 *$ & 78,237 & $19,094^{*}$ \\
\hline VAR*NÍV & 5 & 8,828 & 0,301 & 0,804 & 44,747 & $24,807 *$ \\
\hline DIAC*NÍV & 10 & $18,825^{*}$ & 0,766 & $2,252^{*}$ & 74,439 & $36,023^{*}$ \\
\hline VAR*DIAC*NÍV & 10 & 2,523 & 0,564 & $0,758^{*}$ & 40,435 & $9,398^{*}$ \\
\hline erro 2 & 60 & 7,385 & 0,408 & 0,343 & 52,724 & 14,649 \\
\hline Total corrigido & 107 & & & & & \\
\hline $\mathrm{CV} 1(\%)=$ & 2 & 2,95 & 3,67 & 3,92 & 4,82 & 4,48 \\
\hline $\mathrm{CV} 2(\%)=$ & 1 & 3,18 & 2,63 & 2,79 & 4,21 & 3,64 \\
\hline Média geral: & 2 & 85,38 & 24,31 & 20,98 & 172,39 & 105,09 \\
\hline
\end{tabular}

${ }^{1}$ Variedade (VAR), ${ }^{2}$ período em dias de precipitação mais irrigação até a época de supressão (DIAC), ${ }^{3}$ níveis de adubação (NIV). *Valores significativos estatisticamente $(\mathrm{p}<0,05)$ pelo teste $\mathrm{F}$. 
colheita no meio da safra, o que é interessante para o planejamento das atividades das usinas.

A característica pureza do caldo da cana-de-açúcar está diretamente relacionada com a qualidade da matériaprima, e sofre influência das impurezas minerais e vegetais que são adicionadas à cana no momento da colheita. Verificou-se que a variedade RB85-5453 não diferiu em nenhum tratamento na característica pureza, com valor máximo de 91,59\% no nível 6 aos 195 DIAC, diferindo da variedade SP80-1816, que obteve valores inferiores, atingindo mínimo de 80,41\% no nível 5 aos 195 DIAC (Tabela 3), confirmado na Tabela 4, os resultados demonstraram diferença significativa entre as variedades em estudo. Esses resultados corroboram com os de Duarte Júnior \& Coelho (2008) (90,1\%), Dalri et al. (2008) (92,0\%), e Prado \& Pancelli (2006) (83,8\%).

As normas de qualidade da matéria-prima redigidas pelo Consecana (2006) estabelecem que as unidades industriais só podem recusar o recebimento de carregamentos com pureza abaixo de $75 \%$. Franco (2003) cita que no Estado de São Paulo tem-se, como referência, pureza mínima de $80 \%$ em início de safra e $85 \%$ no transcorrer da safra para que seja recomendada a industrialização da cana.

Na Tabela 6 encontram-se as médias de níveis de adubação dentro de cada DIAC e variedades. Para a variável Pol do caldo, houve diferenças significativas com maiores valores encontrados aos 225 DIAC em ambas as variedades.
Notou-se comportamento crescente no Pol do caldo em relação aos níveis de adubo, com os maiores valores obtidos nos níveis mais elevados de adubação. Constatou-se que os níveis de adubação interferiram na qualidade do caldo, sendo cada um caracterizado por apresentar maior quantidade do elemento potássio e menor de nitrogênio. Esse fato pode ser considerado importante, uma vez que excesso de $\mathrm{N}$ favorece o prolongamento do período vegetativo da cana. Gava et al. (2003) observou que a adubação nitrogenada reduziu o teor de sacarose em quatro variedades de cana-de-açúcar. Segundo Rossetto et al. (2002), a aplicação do nitrogênio aumenta a produtividade agrícola da cultura, todavia decresce a porcentagem de sacarose. Algumas variedades têm comportamento distinto com relação ao nitrogênio, sendo capazes de utilizar mais do que outras, como também em relação ao acúmulo de sacarose; ou seja, altas doses podem não afetar a qualidade dos caldos. A qualidade industrial da cana-de-açúcar irrigada e sob adubação foi estudada no Brasil por diversos autores, dentre eles Carvalho et al. (2008), Dalri et al. (2008), Silva et al. (2009) e Farias et al. (2009).

Dantas Neto et al. (2006), estudando diferentes lâminas totais de irrigação e doses de adubação nitrogênio + potássio, não registraram diferença na qualidade tecnológica da variedade SP 79-1011, sugerindo que a adubação com nitrogênio não interfere na qualidade tecnológica da cana-de-açúcar, mas influencia no primei-

Tabela 2. Médias dos valores de Brix em caldo e ATR no desdobramento do período de irrigação e precipitação (DIAC) com variedades

\begin{tabular}{lcccccc}
\hline & & \multicolumn{2}{c}{ BRIX(\%) } & & \multicolumn{2}{c}{ ATR(\%) } \\
\cline { 3 - 4 } & & SP80-1816 & RB85-5453 & & SP80-1816 & RB85-5453 \\
\hline DIAC & 165 & $22,53 \mathrm{bB}$ & $25,54 \mathrm{aA}$ & & $157,98 \mathrm{bB}$ & $182,47 \mathrm{aA}$ \\
& 195 & $22,89 \mathrm{bB}$ & $25,21 \mathrm{aA}$ & & $159,92 \mathrm{bB}$ & $181,29 \mathrm{aA}$ \\
& 225 & $23,95 \mathrm{bA}$ & $25,71 \mathrm{aA}$ & & $169,55 \mathrm{bA}$ & $183,09 \mathrm{aA}$
\end{tabular}

Médias dentro de cada atributo, seguidas por letras minúsculas, distintas na linha, diferem entre si estatisticamente pelo teste de Scott Knott a $5 \%$ de probabilidade.

Médias dentro de cada atributo, seguidas por letras maiúsculas distintas na coluna diferem entre si estatisticamente pelo teste de Scott Knott a $5 \%$ de probabilidade.

Tabela 3. Médias dos valores de Pureza no desdobramento dos níveis de adubação e períodos de irrigação e precipitação (DIAC)

\begin{tabular}{cccccccc}
\hline \multicolumn{7}{c}{ PUREZA(\%) } \\
\hline & & \multicolumn{7}{c}{ NÍVEIS de ADUBAÇÃo } \\
\cline { 2 - 8 } & & $\mathbf{1}$ & $\mathbf{2}$ & $\mathbf{3}$ & $\mathbf{4}$ & $\mathbf{5}$ & $\mathbf{6}$ \\
\hline \multirow{3}{*}{ DIAC } & 165 & $85,605 \mathrm{aA}$ & $81,63 \mathrm{bB}$ & $86,28 \mathrm{aA}$ & $83,39 \mathrm{bB}$ & $85,17 \mathrm{aA}$ & $87,41 \mathrm{aA}$ \\
& 195 & $84,28 \mathrm{aA}$ & $85,51 \mathrm{aA}$ & $86,32 \mathrm{aA}$ & $85,26 \mathrm{aB}$ & $82,97 \mathrm{aA}$ & $88,46 \mathrm{aA}$ \\
& 225 & $83,17 \mathrm{bA}$ & $84,40 \mathrm{bA}$ & $88,32 \mathrm{aA}$ & $88,14 \mathrm{aA}$ & $85,17 \mathrm{bA}$ & $85,30 \mathrm{bA}$ \\
\hline
\end{tabular}

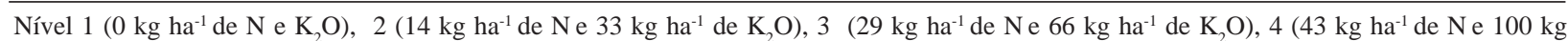
$\mathrm{ha}^{-1}$ de $\mathrm{K}_{2} \mathrm{O}$ ), 5 (57 $\mathrm{kg} \mathrm{ha}^{-1}$ de $\mathrm{Ne} 133 \mathrm{~kg} \mathrm{ha}^{-1}$ de $\mathrm{K}_{2} \mathrm{O}$ ), e 6 (71 kg ha $\mathrm{kg} \mathrm{Ne}^{-1}$ de $\mathrm{kg} \mathrm{ha}^{-1}$ de $\left.\mathrm{K}_{2} \mathrm{O}\right)$.

Médias seguidas por letras minúsculas, distintas na linha, diferem entre si pelo teste de Scott Knott a 5\% de probabilidade.

Médias dentro de cada nível seguidas por letras maiúsculas distintas na coluna diferem entre si pelo teste de Scott Knott a 5\% de probabilidade.

Rev. Ceres, Viçosa, v. 59, n.6, p. 832-840, nov/dez, 2012 
ro estádio fenológico, o vegetativo, assim corroborando com os obtidos por Costa et al. (2003), os quais verificaram que a adubação nitrogenada $\left(100 \mathrm{~kg} \mathrm{ha}^{-1}\right)$ não afetou a qualidade tecnológica da cana-de-açúcar.

Vários trabalhos demonstraram que com excesso de água e nitrogênio a planta não amadurece, correlacionandose positivamente com a umidade e açúcares redutores e negativamente com a sacarose. Prado \& Pancelli (2006) obtiveram resultados na primeira soqueira e observaram que não houve efeito significativo dos diferentes tratamentos de adubação nitrogenada na qualidade tecnológica dos colmos da soqueira de cana. Moura et al. (2005) encontraram valores próximos de Brix e Pol do caldo para diferentes doses de adubação de cobertura de nitrogênio com irrigação, com valores médios de $19,76^{\circ}$ Brix e $17,65 \%$ em Pol do caldo de variedade não relatada.

Tabela 4. Médias dos valores de Pureza em cada variedade de cana de açúcar

\begin{tabular}{lc}
\hline \multicolumn{2}{c}{ PUREZA(\%) } \\
\hline SP80-1816 & $\mathbf{R B 8 5 - 5 4 5 3}$ \\
\hline $83,40 \mathrm{~b}$ & $87,35 \mathrm{a}$ \\
\hline $\begin{array}{l}\text { Médias seguidas por letras minúsculas, distintas na linha, diferem } \\
\text { entre si estatisticamente pelo teste de F a 5\% de probabilidade. }\end{array}$
\end{tabular}

Nesta pesquisa, as variedades mostraram comportamento oposto, o nitrogênio não interferiu na qualidade do caldo pelo fato, principalmente, da quantidade disponibilizada dos diferentes níveis de adubação nitrogenada que se encontraram de baixos valores, de acordo com CFSEMG (1999). No caso de cana irrigada, a maturação deve ser monitorada mediante o controle da aplicação de nitrogênio e da irrigação. A redução da água para controle da irrigação diminui a absorção de nitrogênio pela planta. Em regiões úmidas e quentes, é difícil reduzir a quantidade de água do solo nos períodos de maturação e, consequentemente, a quantidade de nitrogênio aplicada deve ser moderada.

O potássio favoreceu a síntese, o acúmulo e a translocação da sacarose, aumentando a qualidade do caldo das variedades pesquisadas. Verificou-se que as diferenças em qualidade medidas por Pol do caldo dos tratamentos foram influenciadas diretamente pelos diferentes níveis de potássio em combinação com o nitrogênio, uma vez que estava mais disponível para a planta e participava do processo fotossintético, determinante na síntese de açúcares. Sua ação está intimamente associada à natureza catalítica na formação de carboidratos e no desdobramento e na translocação do amido.

Tabela 5. Médias dos valores de porcentagens de Pol do caldo e litros de álcool esperados por tonelada de cana (LITRS ÁLCOOL) no desdobramento do período de irrigação e precipitação (DIAC) com variedades dentro de cada nível de adubação

\begin{tabular}{|c|c|c|c|c|c|}
\hline \multirow{2}{*}{ NÍVEIS } & \multirow{2}{*}{ DIAC } & \multicolumn{2}{|c|}{ POL DO CALDO $(\%)$} & \multicolumn{2}{|c|}{ LTRS DE ÁLCOOL (\%) } \\
\hline & & SP80-1816 & RB85-5453 & SP80-1816 & RB85-5453 \\
\hline \multirow{3}{*}{1} & 165 & $18,87 \mathrm{bB}$ & $21,49 \mathrm{aA}$ & $103,17 \mathrm{bA}$ & $110,21 \mathrm{aA}$ \\
\hline & 195 & $18,88 \mathrm{bB}$ & $21,23 \mathrm{aA}$ & $98,81 \mathrm{bA}$ & $109,96 \mathrm{aA}$ \\
\hline & 225 & $19,81 \mathrm{bA}$ & $21,27 \mathrm{aA}$ & $101,16 \mathrm{aA}$ & $105,22 \mathrm{aA}$ \\
\hline \multirow{3}{*}{2} & 165 & $17,22 \mathrm{bB}$ & $21,81 \mathrm{aA}$ & $90,21 \mathrm{bB}$ & $110,11 \mathrm{aA}$ \\
\hline & 195 & $18,82 \mathrm{bA}$ & $22,35 \mathrm{aA}$ & $98,64 \mathrm{bA}$ & $110,36 \mathrm{aA}$ \\
\hline & 225 & $19,41 \mathrm{bA}$ & $21,56 \mathrm{aA}$ & $101,79 \mathrm{bA}$ & $109,73 \mathrm{aA}$ \\
\hline \multirow{3}{*}{3} & 165 & $19,11 \mathrm{bB}$ & $22,10 \mathrm{aA}$ & $98,98 \mathrm{bB}$ & $110,66 \mathrm{aA}$ \\
\hline & 195 & $19,34 \mathrm{bB}$ & $22,68 \mathrm{aA}$ & $98,88 \mathrm{bB}$ & $110,28 \mathrm{aA}$ \\
\hline & 225 & $20,95 \mathrm{bA}$ & $23,05 \mathrm{aA}$ & $106,64 \mathrm{bA}$ & $113,38 \mathrm{aA}$ \\
\hline \multirow{3}{*}{4} & 165 & $17,79 \mathrm{bC}$ & $21,67 \mathrm{aB}$ & $92,06 \mathrm{bB}$ & $109,14 \mathrm{aA}$ \\
\hline & 195 & $19,51 \mathrm{bB}$ & $22,51 \mathrm{aA}$ & $99,07 \mathrm{bA}$ & $110,51 \mathrm{aA}$ \\
\hline & 225 & 20,99 bA & $23,25 \mathrm{aA}$ & $105,56 \mathrm{bA}$ & $113,44 \mathrm{aA}$ \\
\hline \multirow{3}{*}{5} & 165 & $20,12 \mathrm{bA}$ & $22,06 \mathrm{aA}$ & $99,23 \mathrm{bA}$ & $110,61 \mathrm{aA}$ \\
\hline & 195 & $19,35 \mathrm{bA}$ & $22,10 \mathrm{aA}$ & $96,48 \mathrm{bA}$ & $109,25 \mathrm{aA}$ \\
\hline & 225 & $20,22 \mathrm{bA}$ & $22,69 \mathrm{aA}$ & $102,69 \mathrm{bA}$ & $111,55 \mathrm{aA}$ \\
\hline \multirow{3}{*}{6} & 165 & $19,55 \mathrm{bA}$ & $23,17 \mathrm{aA}$ & $97,06 \mathrm{bA}$ & $115,09 \mathrm{aA}$ \\
\hline & 195 & $19,68 \mathrm{bA}$ & $22,81 \mathrm{aA}$ & $98,59 \mathrm{bA}$ & $112,78 \mathrm{aA}$ \\
\hline & 225 & $20,75 \mathrm{bA}$ & $22,88 \mathrm{aA}$ & $101,31 \mathrm{bA}$ & $110,53 \mathrm{aA}$ \\
\hline
\end{tabular}

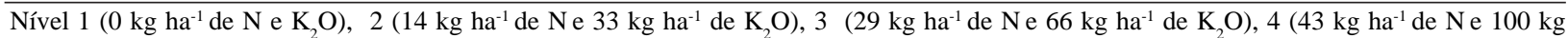
ha $^{-1}$ de $\left.\mathrm{K}_{2} \mathrm{O}\right), 5$ (57 kg ha-1 de Ne $133 \mathrm{~kg} \mathrm{ha}^{-1}$ de $\left.\mathrm{K}_{2} \mathrm{O}\right)$, e $6\left(71 \mathrm{~kg} \mathrm{ha}^{-1}\right.$ de $\mathrm{Ne} 166 \mathrm{~kg} \mathrm{ha}^{-1}$ de $\left.\mathrm{K}_{2} \mathrm{O}\right)$.

Médias dentro de cada atributo, seguidas por letras minúsculas, distintas na linha, diferem entre si estatisticamente pelo teste de Scott Knott a $5 \%$ de probabilidade. Médias dentro de cada nível, seguidas por letras maiúsculas, distintas na coluna, diferem entre si estatisticamente pelo teste de Scott Knott a $5 \%$ de probabilidade. 
Santos et al. (1979) afirmaram que o potássio provocou aumento na produtividade com adubação de até 440 $\mathrm{kg}$ de $\mathrm{K}_{2} \mathrm{O} \mathrm{ha}^{-1}$ e aumentou o teor de açúcar teórico recuperável nos experimentos de campo em algumas regiões do Nordeste.

Muchow et al. (1996), analisando a influência dos nutrientes $\mathrm{N}, \mathrm{P} \mathrm{e} \mathrm{K}$ nas qualidades tecnológicas da cana-deaçúcar, concluíram que, em alguns casos, o nitrogênio, quando aplicado em quantidades inferiores a $50 \mathrm{~kg} \mathrm{ha}^{-1}$, proporcionou efeito depressivo até $120 \mathrm{~kg} \mathrm{ha}^{-1}$ e aumentou a Pol.

ATR (Tabela 2) e litros de álcool (Tabelas 5 e 6) estimado por tonelada de cana obtiveram valores médios 172,389 $\mathrm{kg}$ ATR t-1 e 105,0905 $\mathrm{Lt}^{-1}$ respectivamente.

Dos atributos analisados, o açúcar total recuperável (ATR) é muito importante tanto para a indústria quanto para os produtores, pois em razão dele é que as unidades industriais determinam o preço pago aos produtores, seguindo uma metodologia descrita pela Consecana (2006). Observando-se o parâmetro ATR, verificou-se que a variedade RB85-5453 apresentou melhor resultado, tendo possibilidade de atingir teores superiores a $189,25 \mathrm{~kg}$ de ATR $\mathrm{t}^{-1}$ (Tabela 2). Nos tratamentos de DIAC houve diferenças significativas $(p<0,05)$ apenas aos 165 DIAC nos níveis 2,3 e 4, indicando que maior disponibilidade hídrica favorece o rendimento teórico de açúcar (Tabela 2).

Em todo o Brasil, a cana-de-açúcar tem sido remunerada por seus índices qualitativos, de modo que quanto melhor a qualidade da matéria-prima maior é o preço pago por tonelada de colmos. Todos os índices discutidos nes- te trabalho são usados como base de cálculo para se determinar a quantidade de açúcares totais recuperáveis, expressos em kg de ATR $\mathrm{t}^{-1}$ de cana. Pelos resultados desta pesquisa comprovou-se que a qualidade da matéria-prima pode ser melhorada com a irrigação. Franco (2003), estudando a variedade SP81-3250, utilizando na parcela-testemunha adubação química recomendada, obteve média de 155,89 $\mathrm{kg}$ de ATR t-1 ${ }^{-1}$, sendo a cana-de-açúcar cortada para industrialização no mês de agosto de 2002.

Wiedenfeld (1995), analisando três condições de irrigação ( 95,85 e $65 \%$ da fração de esgotamento do solo) e taxa de adubação média de $136,8 \mathrm{~kg} \mathrm{ha}^{-1}$ de $\mathrm{N}$ para a produção máxima de açúcar, verificou rendimentos de 13,10 e $8 \mathrm{t} \mathrm{ha}^{-1}$ de açúcar, respectivamente. Oliveira et al. (2008), trabalhando com diferentes variedades, obteve maiores valores médios desse indicador encontrados nas variedades RB92579 e RB943365 de 159,97 e 163,88 $\mathrm{kg} \mathrm{t}^{-1}$, respectivamente.

Mais uma vez evidencia-se que os diferentes níveis de adubação não alteraram a qualidade tecnológica das variedades estudadas (Tabela 2). Esses valores estão próximos dos encontrados por Prado \& Pancelli (2006), Duarte Júnior \& Coelho (2008), Dalri et al. (2008), os quais foram de 135,$8 ; 129,0 ;$ e $138,8 \mathrm{~kg}$ de ATR $\mathrm{t}^{-1}$, respectivamente.

Na característica litros de álcool estimado por tonelada de cana, procedeu-se correlação dessa com os percentuais de sacarose da planta medidos em Brix, Pol e o processo produtivo na fermentação referenciada de $85 \%$ em rendimento. Nas Tabelas 5 e 6 evidencia-se que a vari-

Tabela 6. Médias dos valores de Pol do caldo e litros de álcool esperado por tonelada de cana (LITRS ALCOOL), interagindo cada nível de adubação dentro dos períodos (dias de irrigação mais precipitação após corte DIAC) e variedades

\begin{tabular}{|c|c|c|c|c|c|c|c|}
\hline \multirow{2}{*}{ VAR } & \multirow{2}{*}{ DIAC } & \multicolumn{6}{|c|}{ NÍVEIS } \\
\hline & & 1 & 2 & 3 & 4 & 5 & 6 \\
\hline & & \multicolumn{6}{|c|}{ POL DO CALDO (\%) } \\
\hline \multirow{3}{*}{ SP80-1816 } & 165 & $18,87 \mathrm{~b}$ & $17,22 \mathrm{c}$ & $19,11 \mathrm{~b}$ & $17,79 \mathrm{c}$ & $20,12 \mathrm{a}$ & $19,55 \mathrm{a}$ \\
\hline & 195 & $18,88 \mathrm{~b}$ & $18,82 b$ & $19,34 \mathrm{a}$ & $19,51 \mathrm{a}$ & $19,35 \mathrm{a}$ & $19,68 \mathrm{a}$ \\
\hline & 225 & $19,81 b$ & $19,41 b$ & $20,95 \mathrm{a}$ & $20,99 a$ & $20,22 \mathrm{a}$ & $20,75 a$ \\
\hline \multirow{4}{*}{ RB85-5453 } & 165 & $21,49 b$ & $21,81 b$ & $22,10 \mathrm{~b}$ & $21,67 b$ & $22,06 b$ & $23,17 \mathrm{a}$ \\
\hline & 195 & $21,23 b$ & $22,35 \mathrm{a}$ & $22,68 \mathrm{a}$ & $22,51 \mathrm{a}$ & $22,10 \mathrm{a}$ & $22,81 \mathrm{a}$ \\
\hline & 225 & $22,27 b$ & $21,56 b$ & $23,05 \mathrm{a}$ & $23,25 \mathrm{a}$ & $22,69 \mathrm{a}$ & $22,88 \mathrm{a}$ \\
\hline & & \multicolumn{6}{|c|}{ LTRS ÁLCOOL $\left(\mathrm{L} \mathrm{t}^{-1}\right)$} \\
\hline \multirow{3}{*}{ SP80-1816 } & 165 & $103,17 \mathrm{a}$ & $90,21 b$ & $98,98 \mathrm{a}$ & $92,06 b$ & $99,23 \mathrm{a}$ & $97,06 \mathrm{a}$ \\
\hline & 195 & $98,81 \mathrm{a}$ & $98,64 \mathrm{a}$ & $98,88 \mathrm{a}$ & $99,07 \mathrm{a}$ & $96,48 \mathrm{a}$ & $98,59 \mathrm{a}$ \\
\hline & 225 & $101,16 \mathrm{a}$ & $101,79 \mathrm{a}$ & $106,64 \mathrm{a}$ & $105,56 \mathrm{a}$ & $102,69 a$ & $101,31 a$ \\
\hline \multirow{3}{*}{ RB85-5453 } & 165 & $110,21 \mathrm{a}$ & $110,11 \mathrm{a}$ & $110,66 a$ & $109,14 \mathrm{a}$ & $110,61 \mathrm{a}$ & $115,09 \mathrm{a}$ \\
\hline & 195 & $109,96 a$ & $110,36 a$ & $110,28 \mathrm{a}$ & $110,51 \mathrm{a}$ & $109,25 \mathrm{a}$ & $112,78 \mathrm{a}$ \\
\hline & 225 & $105,22 \mathrm{a}$ & $109,73 a$ & $113,38 \mathrm{a}$ & $113,44 \mathrm{a}$ & $111,55 \mathrm{a}$ & $110,53 a$ \\
\hline
\end{tabular}

Nível 1 (0 $\mathrm{kg} \mathrm{ha}^{-1}$ de $\mathrm{N}$ e $\left.\mathrm{K}_{2} \mathrm{O}\right), 2$ (14 $\mathrm{kg} \mathrm{ha}^{-1}$ de $\mathrm{Ne} 33 \mathrm{~kg} \mathrm{ha}^{-1}$ de $\left.\mathrm{K}_{2} \mathrm{O}\right), 3\left(29 \mathrm{~kg} \mathrm{ha}^{-1}\right.$ de Ne $66 \mathrm{~kg} \mathrm{ha}^{-1}$ de $\left.\mathrm{K}_{2} \mathrm{O}\right), 4(43 \mathrm{~kg}$ ha-1 de Ne $100 \mathrm{~kg}$ ha $^{-1}$ de $\left.\mathrm{K}_{2} \mathrm{O}\right), 5\left(57 \mathrm{~kg} \mathrm{ha}^{-1}\right.$ de $\mathrm{Ne} 133 \mathrm{~kg} \mathrm{ha}^{-1}$ de $\left.\mathrm{K}_{2} \mathrm{O}\right)$, e $6\left(71 \mathrm{~kg} \mathrm{ha}^{-1}\right.$ de $\mathrm{Ne} 166 \mathrm{~kg} \mathrm{ha}^{-1}$ de $\left.\mathrm{K}_{2} \mathrm{O}\right)$.

Médias dentro de cada atributo, seguidas por letras minúsculas, distintas na linha, diferem entre si estatisticamente pelo teste de Scott Knott a $5 \%$ de probabilidade.

Rev. Ceres, Viçosa, v. 59, n.6, p. 832-840, nov/dez, 2012 
edade RB85-5453 teve melhor desempenho em litros de álcool estimados, que pode ser explicado pelo seu comportamento de maturidade precoce e consequente maior acúmulo de sacarose no seu colmo no período de safra pesquisado. Registraram-se valores máximos de 115,09 L $\mathrm{t}^{-1}$ no nível 6 aos 165 DIAC da variedade RB85-5453, e mínimo de 90,21 L t ${ }^{-1}$ no nível 2 aos 165 DIAC da variedade SP80-1816. O alto rendimento encontrado na variedade RB85-5453 dá indícios de que boas nutrições minerais e condição hídrica no desenvolvimento, associadas a um período considerável de restrição hídrica, trarão bons rendimentos no processo fermentativo dessas variedades estudadas.

Dantas Neto et al. (2006), trabalhando com diferentes lâminas de irrigação e doses de adubação, constataram rendimento bruto de álcool $\mathrm{em} \mathrm{m}^{3} \mathrm{ha}^{-1}$, valores significativos ao parâmetro adubação, com média de $6,25 \mathrm{~m}^{3} \mathrm{ha}^{-1}$ na adubação mínima de $86 \mathrm{~kg} \mathrm{ha}^{-1}$ de $\mathrm{N}$ e $8,91 \mathrm{~m}^{3} \mathrm{ha}^{-1}$ na adubação máxima de $305 \mathrm{~kg} \mathrm{ha}^{-1}$ de $\mathrm{N}$. Essa característica é de grande importância, pois estima a produtividade do canavial em álcool, tendo referência da qualidade da cana processada.

O aumento do rendimento em álcool com a aplicação de nitrogênio também foi observado por Carvalho et al. (2009), os quais verificaram que a aplicação de $112 \mathrm{~kg}$ ha ${ }^{1}$ de nitrogênio proporcionou o maior rendimento de 9,8 $\mathrm{m}^{3}$ ha $^{-1}$ de álcool. Nesse sentido, observa-se que os resultados encontrados neste trabalho ratificam o obtido por Silva et al. (2003) $\left(10,5 \mathrm{~m}^{3} \mathrm{ha}^{-1}\right)$, possivelmente em função do ambiente de cultivo, da produtividade e da variedade ser em diferentes dos do presente trabalho.

\section{CONCLUSÕES}

O período de 225 DIAC foi o melhor em desempenho, porém com valores próximos em qualidade tecnológica nas demais supressões na região pesquisada.

O nível 6 (71 kg ha-1 de $\mathrm{Ne} 166 \mathrm{~kg} \mathrm{ha}^{-1}$ de $\left.\mathrm{K}_{2} \mathrm{O}\right)$ de adubação propiciou melhor desempenho nas condições de realização deste trabalho.

As variedades RB85-5453 e SP80-1816 são promissoras para a região Norte do Estado de Minas Gerais, tendo boa adaptação às condições edafoclimáticas, porém a variedade RB85-5453 foi a mais indicada para a região nas condições de realização deste trabalho, assim atendendo às exigências de produção sucroalcooleira no seu terceiro ciclo de produtividade.

\section{REFERÊNCIAS}

Araújo NC (2006) Cana-de-açúcar: resposta técnica. Produzida pelo Serviço Brasileiro de Respostas Técnicas. 7p. Disponível em: <http://www.agrobyte.com.br/cana.htm>. Acessado em: 03 de Setembro de 2010.
Bernardo S, Soares AA \& Mantovane EC (2005) Manual de irrigação, $7^{\mathrm{a}}$ ed. Viçosa, UFV. 611p.

Carvalho CM, Azevedo HM, Dantas Neto J, Melo EP, Silva CTS \& Gomes Filho RR (2008) Resposta dos parâmetros tecnológicos da terceira folha da cana-de-açúcar a diferentes níveis de irrigação. Revista Brasileira de Ciências Agrárias, 3:337-342.

Carvalho CM, Azeredo HM, Neto JD, Farias CH, Silva CTS \& Gomes Filho RR (2009) Rendimento de açúcar e álcool da canade-açúcar submetida a diferentes níveis de irrigação. Revista Brasileira de Ciências Agrárias, 4:72-77.

Conselho dos Produtores de Cana-de-Açúcar, Açúcar e Álcool do Estado de São Paulo - CONSECANA (2006) Manual de Instruções. $5^{\circ}$ ed. Piracicaba-SP, Consecana-SP. 112p.

Comissão de fertilidade de solo do Estado de Minas Gerais - CFSEMG (1999) Recomendações para o uso de corretivos e fertilizantes em Minas Gerais - 5a aproximação. Lavras, CFSEMG. 359p.

Costa MCG, Vitti GC \& Cantarella H (2003) Volatilização de N-NH3 de fontes nitrogenadas em cana-de-açúcar colhida sem despalha a fogo. Revista Brasileira de Ciência do Solo, 27:631-637.

Dalri AB, Cruz RL, Garcia CJB \& Duenhas LH (2008) Irrigação por gotejamento subsuperficial na produtividade e qualidade de cana-de-açúcar. Revista Irriga, 13:01-11.

Dantas Neto J, Figueredo JL da C, Farias CH de A, Azevedo HM de \& Azevedo CAV de (2006) Resposta de cana-de-açúcar, primeira soca, a níveis de irrigação e adubação de cobertura. Revista Brasileira de Engenharia Agrícola e Ambiental, 10:283-288.

Duarte Júnior J \& Coelho FC (2008) A cana-de-açúcar em sistema de plantio direto comparado ao sistema convencional com e sem adubação. Revista Brasileira de Engenharia Agrícola e Ambiental, 12:576-583.

Empresa Brasileira de Pesquisa Agropecuária - Embrapa (1999) Centro Nacional de Pesquisa de Solos. Sistema Brasileiro de Classificação de Solos. Brasília, Embrapa. 412p.

Farias CHA, Fernandes PD, Gheyi HR \& Dantas Neto J (2009) Qualidade industrial de cana-de-açúcar sob irrigação e adubação com zinco, em Tabuleiro Costeiro paraibano. Revista Brasileira de Engenharia Agrícola e Ambiental, 13:419-428.

Ferreira DF (2000) Manual do sistema Sisvar para análises estatísticas. Lavras, UFLA. 66p.

Fernandes AC (2000) Cálculos na agroindústria da cana-de-açúcar. Piracicaba, STAB: Açúcar, Álcool e Subprodutos. 193p.

Franco A (2003) Cana-de-açúcar cultivada em solo adubado com lodo de esgoto e vinhaça: nitrogênio no sistema solo-planta, produtividade e características tecnológicas. Dissertação de Mestrado. Universidade Estadual Paulista, Jaboticabal. 90p.

Gava GJ, Trivelin PCO, Vitti AC \& Oliveira MW (2003) Recuperação do nitrogênio $(\mathrm{N})$ da uréia e da palhada por soqueira de cana-de-açúcar (Saccharum spp.). Revista Brasileira de Ciência do Solo, 27:621-630.

Moura MVP da S, Farias CH de A, Azevedo CAV de, Dantas Neto J, Azevedo H M de \& Pordeus RV (2005) Doses de adubação nitrogenada e potássica em cobertura na cultura da cana-deaçúcar, primeira soca, com e sem irrigação. Ciência e Agrotecnologia, 29:753-760.

Muchow RC, Robertson MJ \& Wood AW (1996) Effect of nitrogen on the time course of sucrose accumulation in sugar cane. Field Crops Research, 7:143-153.

Prado R de M \& Pancelli MA (2006) Nutrição em soqueira e a qualidade tecnológica da cana-de-açúcar. STAB: Açúcar, Álcool e Subprodutos, 25:60-63.

Rodrigues JD (1995) Fisiologia da cana de açúcar. Botucatu, UNESP. $101 \mathrm{p}$. 
Rossetto R, Farhat M, Furlan R, Gil MA \& Silva SF (2002) Eficiência agronômica do fosfato natural na cultura da cana-deaçúcar. In: Congresso Nacional da Sociedade dos Técnicos Açucareiro e Alcooleiros do Brasil, Pernambuco. Anais, STAB. p. $276-282$.

Santos MAC dos, Sobral AF de, Cordeiro DA \& Araújo JDL de (1979) Adubação da cana-de-açúcar: resumo informativo. Carpina, IAA/PLANALSUCAR. 3p.

Silva M de A, Carlin SD, Campana MP, Landell MGA, Perecin D \& Vasconcelos ACM (2003) Brotação da cana-de-açúcar em condições de casa de vegetação. STAB: Açúcar Álcool e Subprodutos, 22:28-31.
Silva AB, Dantas Neto J, Farias CHA, Azevedo CAV, Azevedo HM (2009) Rendimento e qualidade da cana-de-açúcar irrigada sob adubações de nitrogênio e potássio em cobertura. Revista Caatinga, 22:236-241.

Wiedenfeld RP (1995) Effects of irrigation and $\mathrm{N}$ fertilizer application on sugar cane yield and quality. Field Crop Research, 43:101-108.

Wiedenfeld RP (2000) Water stress during different sugarcane growth periods on yield and response to $\mathrm{N}$ fertilizer. Agricultural Water Management, 43:173-182. 